Danilo Florentino PEREIRA ${ }^{1}$ Fábio Penna Firme CURTO² Irenilza de Alencar N $\ddot{A} \ddot{A} S^{3}$

Correspondência para:

DANILOFLORENTINO PEREIRA

Campus de Tupã

Universidade Estadual Paulista

Av. Domingos da Costa Lopes, 780, Jardim

17603-660 - Tupã-SP

danilo@tupa.unesp.br

Recebido para publicação: 09/06/2005 Aprovado para publicação: 21/09/2005

\title{
Diferenças nos comportamentos individuais quanto à preferência de uso de locais de matrizes pesadas em função do ambiente térmico
}

1 - Campus de Tupã da Universidade Estadual Paulista, Tupã - SP

2 - Faculdades Fleming, Campinas - SP

3 - Faculdade de Engenharia Agrícola da Universidade de Campinas, Campinas - SP

\section{Resumo}

O estudo do comportamento tem se mostrado eficiente na identificação do bem-estar de aves alojadas. As identificações de preferências do grupo quanto o ambiente construtivo e o ambiente térmico vem sendo estudado em diversos países. Todavia, a habilidade dos homeotermos de se aclimatarem em ambientes inóspitos, resulta em necessidades ambientais diferentes para cada indivíduo, culminando em uma heterogeneidade no lote e inevitáveis perdas na produção. Para a diminuição dessas perdas, é necessário que se conheça as necessidades de cada indivíduo alojado para que grupos com necessidades semelhantes sejam formados. Este trabalho teve como objetivo demonstrar a viabilidade da utilização da telemetria e identificação eletrônica para o monitoramento individual de matrizes pesadas e identificar as diferenças comportamentais nas aves alojadas, em modelos de escala reduzida e distorcida. Os resultados demonstraram que a telemetria e a identificação eletrônica foram eficientes na identificação do bem-estar das matrizes pesadas e, através da análise observacional de gráficos de distribuição de freqüências, foi possível identificar os indivíduos que melhor se adaptaram ao ambiente térmico do alojamento.

\section{Introdução}

A identificação do bem-estar de aves tem sido objeto de estudo de muitos pesquisadores. Zhou ${ }^{1}$ e Zhou, Fugita e Yamamoto $^{2}$. estudando o efeito do ambiente térmico na viscosidade do sangue de frangos de corte, descreveram que a viscosidade do sangue diminui quando as aves são expostas a uma condição de temperatura acima da zona termoneutra. A importância do balanço ácido-base foi estudada por Teeter e Belay ${ }^{3}$, que concluíram que este balanço é afetado pelas condições de temperatura, sendo este um fator que interfere diretamente no crescimento das aves.

Os estudos feitos para a determinação do estresse de aves, baseados em respostas fisiológicas, são eficientes e precisos. Todavia, pesquisadores vêm estudando o comportamento de maneira a identificar, mesmo que indiretamente, estados de estresse em aves alojadas. Pettit-Riley e Estevez ${ }^{4}$ estudaram os efeitos do crescimento e do acesso a poleiros no comportamento agressivo de frangos de corte, observando que o comportamento agressivo aumenta de acordo com o nível do crescimento e a densidade de aves no poleiro ${ }^{5}$.

A complexidade do ambiente construtivo, em termos do uso dos espaços internos, foi estudada por María, Escós e Alados ${ }^{6}$, onde os autores demonstraram que as atividades locomotoras diminuem com o aumento do estresse das aves. Bizeray et al. ${ }^{7}$ estudaram os efeitos da utilização dos espaços do alojamento, o ambiente e a atividade física de frangos de corte, concluíram que a inclusão de barreiras no 
ambiente, proporcionam a expressão de "comportamentos naturais" como o empoleirar, aumentando o bem-estar das aves e previne problemas de saúde, como patologias locomotoras e laminite ${ }^{8}$.

A aplicação de tecnologias de rádiofreqüência para o monitoramento das atividades físicas visando o conhecimento das respostas fisiológicas de aves vem sendo estudada em vários países. Korthals, McDonald e Elgenberg', apresentaram um trabalho demonstrando suas experiências com transponders para o monitoramento de respostas bioenergéticas. Kettlewell, Mitchell e Meeks ${ }^{10}$ demonstraram a viabilidade do monitoramento da freqüência cardíaca e da temperatura interna corporal de aves em laboratório. A tecnologia consistia no implante de sensores nas aves, através de cirurgia, e a transmissão de dados ocorreu a uma distância de até dez metros. Curto ${ }^{11}$ descreveu a utilização de identificadores eletrônicos para a identificação e monitoramento de indivíduos em uma população.

O objetivo deste trabalho foi demonstrar, através de monitoramento digital utilizando radio-freqüência, que indivíduos (matrizes pesadas) pertencentes a um grupo, têm preferências térmicas diferentes, quando observados os comportamentos de uso de determinado espaço físico.

\section{Materiais e Métodos}

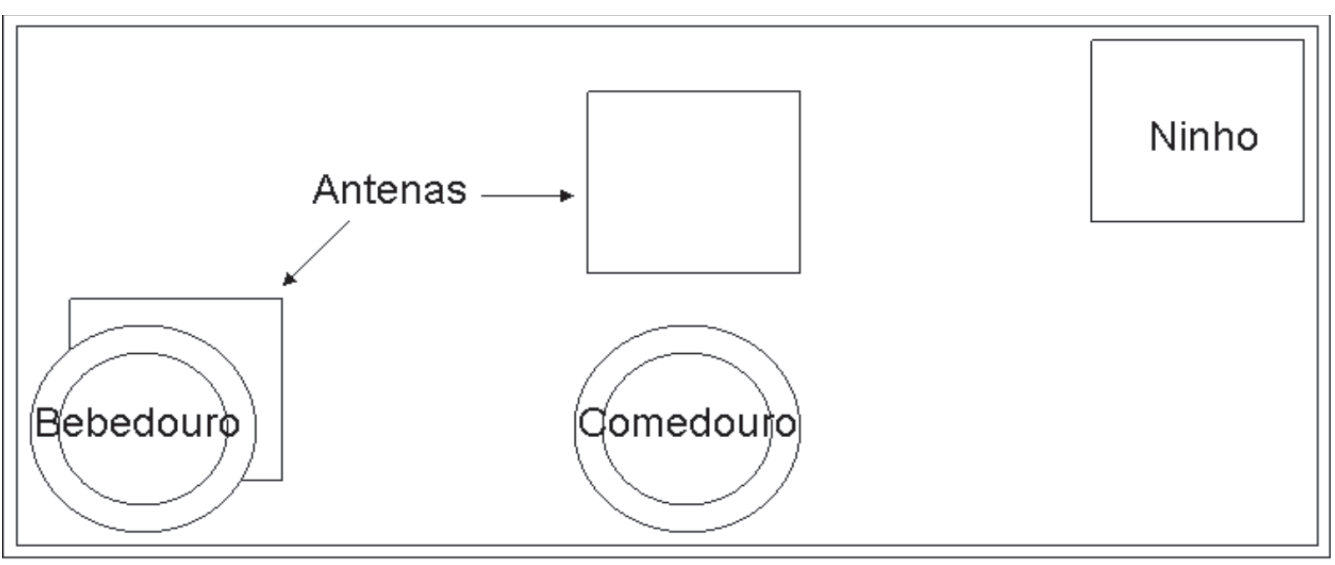

O experimento foi conduzido no município de Campinas, SP situada à latitude de $22^{\circ} 53^{\prime} \mathrm{S}$, altitude de $695 \mathrm{~m}$, tipo climático Cwa, segundo a classificação de Köeppen, sendo caracterizado como clima tropical de altitude com inverno seco e verão quente, na área experimental do Laboratório de Conforto Ambiental da Faculdade de Engenharia Agrícola da UNICAMP, em galpão em escala reduzida e distorcida, com orientação leste-oeste, que reproduz as condições de um galpão de produção.

Para as medidas de dados de ambiente, foram utilizados termopares do tipo J, instalados a uma altura de um metro do piso, acoplados a um datalogger, que enviava as informações em tempo real para um computador. Foram monitorados os comportamentos de uso do bebedouro e da região livre entre o comedouro e a parede norte, denominada passagem, utilizando-se a tecnologia de Identificação Eletrônica. Os registros de comportamentos de uso destes locais foram feitos em tempo real e correlacionados com as condições do ambiente, através de um software desenvolvido para gerenciar este experimento. A figura 1 mostra o esquema da montagem experimental.

Oito matrizes pesadas (frango de corte) da linhagem Hybro-G foram identificadas eletronicamente. Os implantes foram feitos entre o pé e a coxa das aves, conforme proposto por Pereira et al. ${ }^{12}$.

Figura 1- Esquema da montagem experimental 
Foram analisados os dados coletados entre outubro/2000 e janeiro/2001, coincidindo com o verão na região. Utilizou-se, para a identificação das diferentes respostas de freqüências de uso, a análise estatística descritiva e teste de homogeneidade. Para a verificação das diferenças quanto às preferências térmicas no uso dos locais, os dados de temperatura do ar (TBS) e a umidade relativa (UR) foram divididos em classes e gráficos de contorno foram gerados, demonstrando visualmente as diferenças. O programa computacional Minitab ${ }^{\circledR}$ foi utilizado para as análises estatísticas.

\section{Resultados e Discussão}

Nos dados coletados, foi aplicado o teste de homogeneidade para as variáveis, buscando verificar se, em números absolutos de freqüência de uso dos locais monitorados, os indivíduos se comportaram de maneira diferente. Rejeitou-se a hipótese, ao nível de significância de 5\%, da variância das freqüências serem iguais no bebedouro, prevalecendo a diferença entre as aves quanto à utilização deste local (Figura 2). Por outro lado, não se rejeitou a hipótese, ao nível de significância de 5\%, da variância das freqüências serem iguais na passagem (Figura $3)$.

Todavia, este teste avalia apenas os valores globais de utilização, sem levar em conta as freqüências de uso em função das variações do ambiente. Para identificar as diferenças quanto às variações do ambiente, os valores registrados de temperatura do ar (TBS) e umidade relativa (UR) foram divididos em classes, e foram gerados gráficos de superfície para cada ave monitorada. As figuras 4 e 5 mostram a distribuição da freqüência de comportamento de uso de duas aves, com-parativamente, para o bebedouro e para a passagem, respectivamente.

Observa-se que os comportamentos de uso dos locais em função do ambiente são diferentes entre as aves. No bebedouro

Teste de homogeneidade para a variância no bebedouro



Figura 2 - Resultado do teste de homogeneidade para a variância dos dados individuais de cada ave durante o uso do bebedouro 
Teste de homogeneidade da variância para a passagem

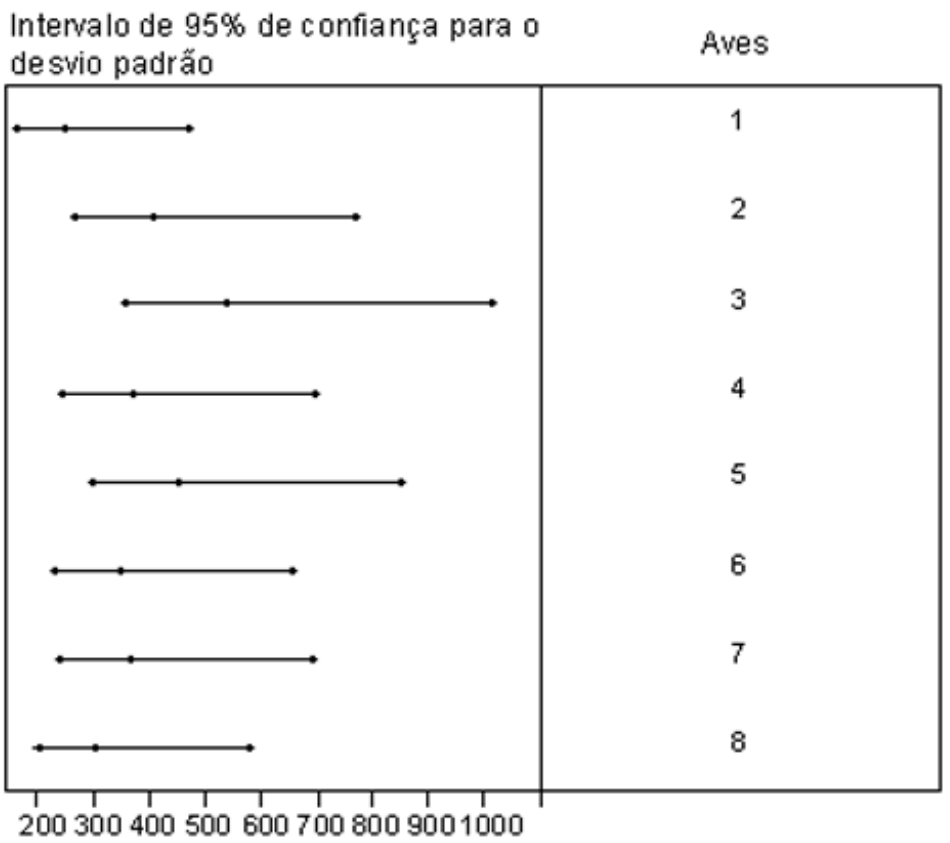

Barlett Test P Valor $=0,136$

Figura 3 - Resultado do teste de homogeneidade para a variância dos dados individuais de cada ave durante o uso da passagem

(Figura 4), as distribuições das freqüências de uso do local são semelhantes para algumas aves, porém, o número absoluto da freqüência de uso é diferente. Esta observação vai de encontro com os resultados observados no teste de homogeneidade (Figura 2), onde já se havia constatado diferença estatística nas freqüências absolutas $(\mathrm{P}<0,01)$.

Analisando as distribuições de freqüência no bebedouro, observa-se que existem duas ilhas de maior concentração de uso, que são comuns a todas as aves. A ilha delimitada pelas altas temperaturas e baixas umidades relativas reflete o desconforto das matrizes neste ambiente, que buscam com maior freqüência o microclima gerado em torno do bebedouro. Cabe ressaltar que o experimento foi conduzido em condições de campo, ou seja, as condições ambientais ocorreram em períodos de tempo curtos. Shinder et al. demonstraram o aumento no estresse de frangos de corte quando expostos a temperaturas baixas durante períodos curtos e repetidos de tempo.

A figura 5 mostra as distribuições das freqüências de uso da passagem para as aves monitoradas. Observa-se que, apesar do teste de homogeneidade para este local (Figura 3) não ter verificado diferença estatística significativa $(\mathrm{P}>0,13)$, as distribuições das freqüências em relação ao ambiente são visualmente diferentes.

A passagem constitui um local onde se procurou monitorar a movimentação das aves dentro do galpão. Observa-se na figura 5 , que o uso deste local foi decrescendo com o aumento da temperatura do ar (TBS). Segundo Rutz $^{13}$, quando o ambiente térmico encontrase acima da zona de termoneutralidade, as aves diminuem as atividades físicas, a fim de diminuir a produção interna de calor, corroborando com os resultados observados na figura 5 . Estes resultados estão de acordo com Bizeray et al. ${ }^{7}$ e María, Escós e Alados 6 que identificaram uma diminuição nas atividades físicas com o aumento do estresse térmico em aves alojadas. 


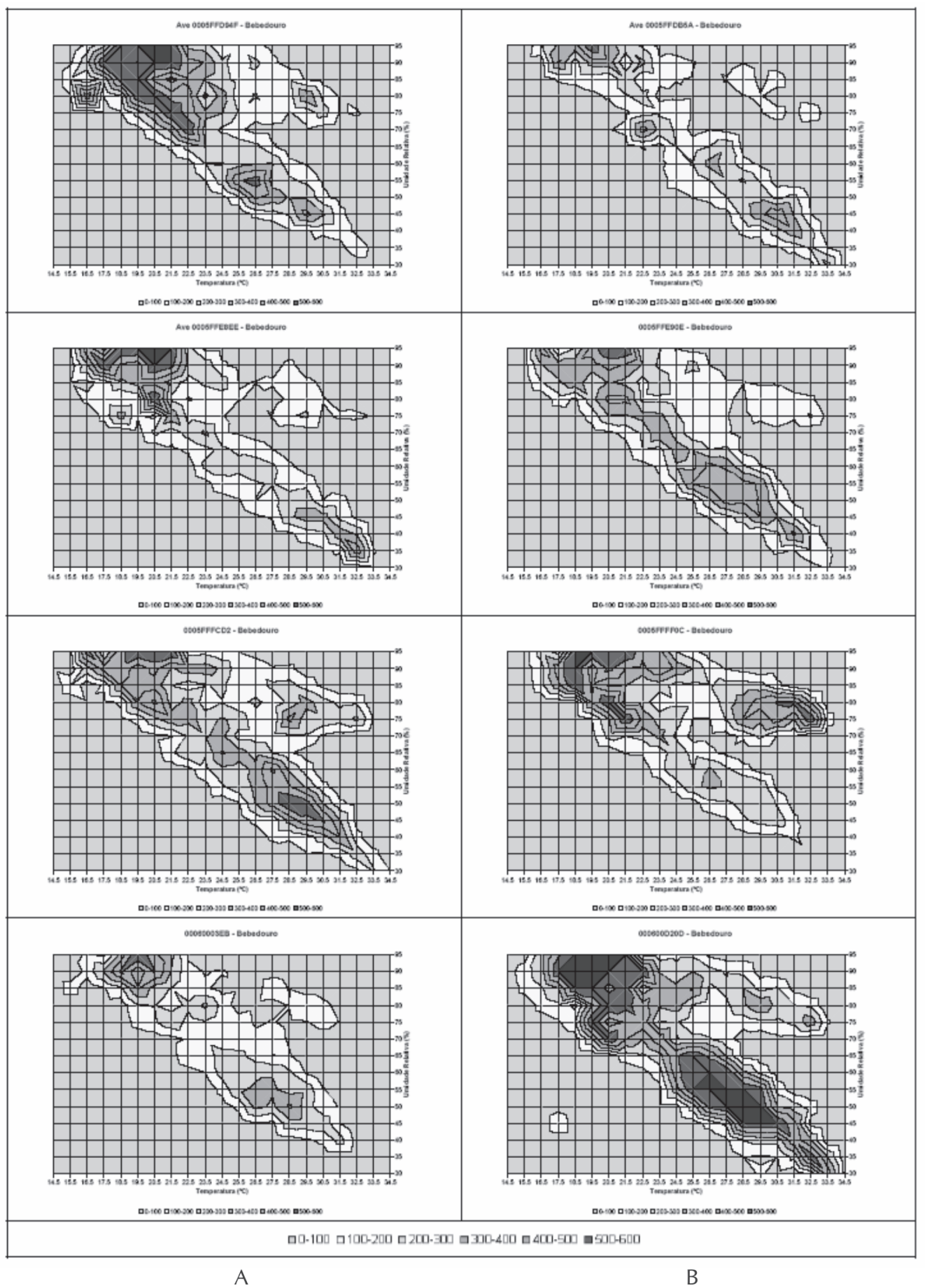

Figura 4-Comparação do comportamento de uso do bebedouro para duas aves (A e B), através de mapas de distribuição de freqüências 


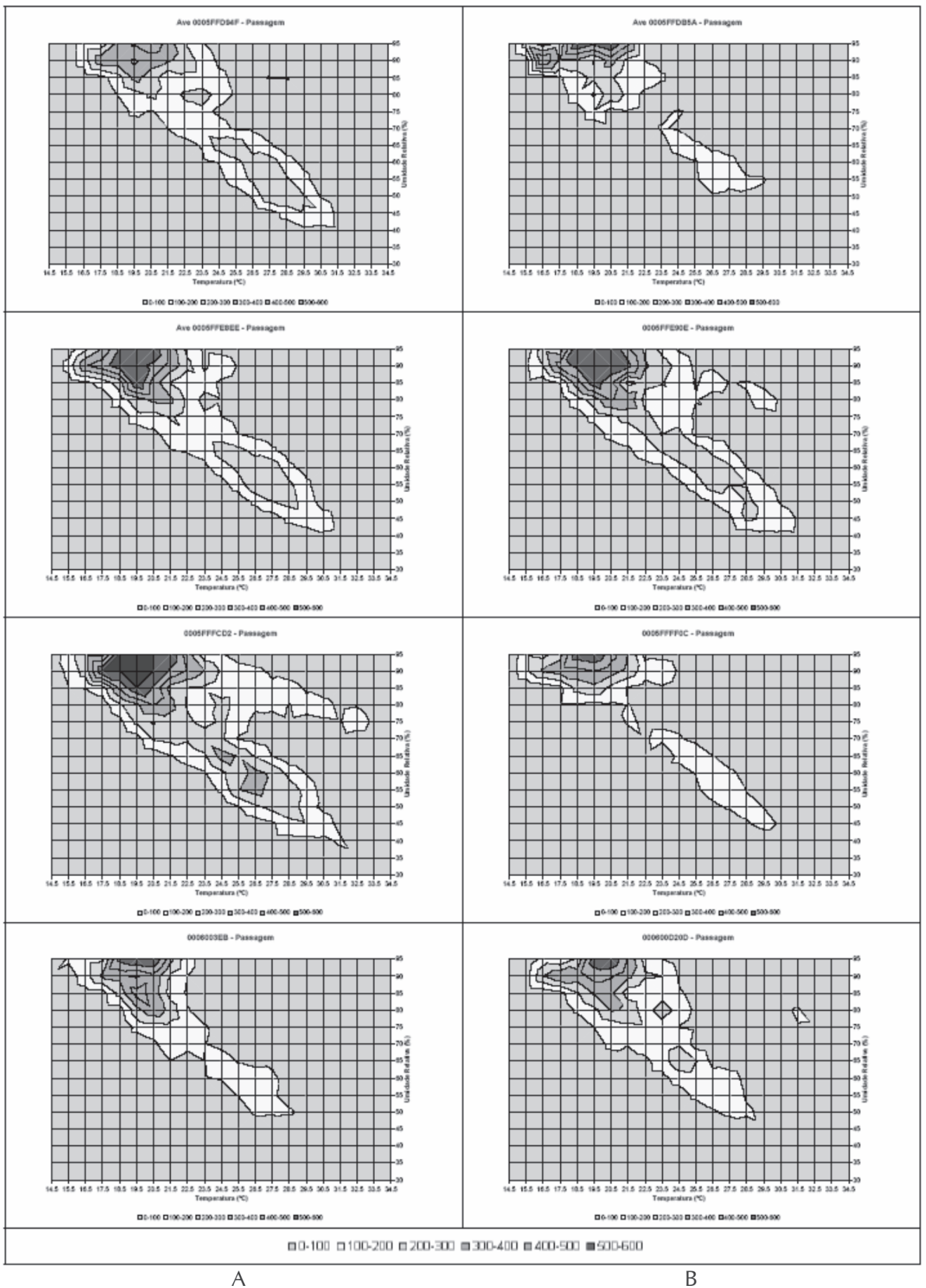

Figura 5 - Comparação do comportamento de uso da passagem para duas aves (A e B), através de mapas de distribuição de freqüências 


\section{Conclusões}

O uso da tecnologia de identificação eletrônica no monitoramento dos comportamentos de uso de locais permitiu identificar diferenças na freqüência de uso, entre indivíduos de um grupo de matrizes pesadas. A visualização das diferentes preferências de uso de locais pelos indivíduos permitiu identificar os indivíduos que se adaptaram melhor ao ambiente experimental.

Os gráficos de contorno mostram as freqüências de utilização de cada local monitorado em função das condições ambientais registradas. Verificou-se, a partir da análise destes gráficos, que o comportamento grupal das aves no bebedouro se concentrou em condições de temperatura baixa e umidade relativa alta e temperatura alta e umidade relativa baixa. $\mathrm{Na}$ análise das freqüências de uso da passagem, observou-se uma redução da atividade física nas condições de temperatura alta, corroborando com os resultados publicados por outros autores.

\section{Agradecimentos}

Os autores agradecem à FAPESP e ao Prodetab pela subvenção da pesquisa.

\title{
Differences in the individual behavior as related to the preference of use of espaces of broiler breeders as a function of the thermal environment
}

\begin{abstract}
The study of animal behavior has been efficient in identifying welfare of housed broilers. Bird preference related to housing and thermal environment has been studied in several countries. However the ability of homeotherms to adept in hostile ambient results in distinct individual needs, and leads to heterogeneity in the flock and inevitable loss in production. To reduce these losses it is necessary to know the individual needs of group of birds in order to group the similar individuals together. This research had the objective to demonstrate the feasibility of use of telemetry and electronic identification for monitoring the individual preferences and specific behavior of female broiler breeders in reduced and distorted scale model. The results showed that the use of electronic identification associated to telemetry were efficient in studying the movement of birds. Through the observational analyses of obtained frequency distribution graphs it was possible to identify the individuals that better adapted to specific housing thermal environment.
\end{abstract}

\section{Referências}

1 ZHOU, W. T.; FUGITA, M. YAMAMOTO, S. Effects of ambient temperatures on blood viscosity and plasma protein concentration of broiler chickens (Gallus domesticus) Journal of Thermal Biology, v. 24, p. 105112, 1999a.

2 ZHOU, W. T. et al. Distribution of body fluid and change of blood viscosity in broiler (Gallus domesticus) under high temperature exposure. Journal of Thermal Biology, v. 24, p. 193-197, 1999b.

3 TEETER, R. G.; BELAY, T. Broiler management during acute heat stress. Animal Feed Science and Technology, v. 58, p. 127-142, 1996.
Key-words:

Female broiler

breeder.

Telemetry.

Behavior.
4 PETTIT-RILEY, R; ESTEVEZ, I. Effects of density on perching behaviour of broiler chickens. Applied Animal Behaviour Science, v. 71, p. 127-140, 2001.

5 PETTIT-RILEY, R.; ESTEVEZ, I.; RUSSEK-COHEN, E. Effects of crowding and access to perches on aggressive behaviour in broiler. Applied Animal Behaviour Science, v. 79, p. 11-25, 2002.

6 MARÍA, G. A.; , ESCÓS, J.; ALADOS, C . L. Complexity of behavioural sequences and their relation to stress conditions in chickens (Gallus gallus domesticus): a non-invasive technique to evaluate animal welfare. Applied Animal Behaviour Science, v. 86, p. 93-104, 2004.

7 BIZERAY, D. et al. ESTEVEZ, I, LETERRIER, C, FAURE, J M. Effects of increasing environmental 
complexity on the physical activity of broiler chickens. Applied Animal Behaviour Science, v. 79, p. 27-41, 2002.

8 WEEKS, C. A.; The behaviour of broiler chickens and its modification by lameness. Applied Animal Behaviour Science, v. 67, 2000, p. 111-125.

9 KORTHALS, R. L.; McDONALD, T. P.; EIGENBERG, R. A. Experiences with Transponders for Monitoring Bioenergetic Responses. ASAE meeting presentation. Paper no. 923010. Charlotte, North Carolina, 1992.

10 KETTLEWELL, P. J., MITCHELL, M. A, MEEKS, I. R. An implanteble radio-telemetry system for remote monitoring of heart rate and deep body temperature in poultry, Computers and Electronics in Agriculture, v. 17, p. $161-175,1997$.

11 CURTO, F. P. F. Desenvolvimento de um sistema de identificação eletrônica para auxílio no gerenciamento de informações na área de produção animal. 1998, 102 f. Dissertação (Mestrado) - Instituto de Informática, PUC Campinas, Campinas 1998.

12 PEREIRA, D. F., et al. Avaliação do local do implante de microchip utilizados para identificação eletrônica em matrizes pesadas (Evaluating the place for transponder implant used in EID for broiler's breeder). Suplement of Brazilian Journal of Poultry Science. v. 1, p. 53, 2001.

13 RUTZ, F. Aspectos fisiológicos que regulam o conforto térmico as aves. In: CONFERÊNCIA APINCO, 1994, Campinas. Anais. Campinas, p. 99-110. 